\title{
La tomographie optique cohérente pour l'étude des instruments de musique vernis
}

La technique d'imagerie à trois dimensions qu'est la tomographie par cohérence optique (OCT) est reconnue dans le domaine biomédical, mais elle présente également de nombreux intérêts pour l'étude des objets du patrimoine : cette méthode d'analyse sans prélèvement ni contact appliquée au vernis des instruments de musique permet d'obtenir des coupes virtuelles et apporte ainsi des informations précieuses pour mieux comprendre les anciennes techniques de vernissage.

\section{$>$ Gaël LATOUR}

Institut des Nanosciences de Paris puis Institut Fresnel, Marseille (actuellement au Laboratoire d'optique et biosciences, Ecole Polytechnique, Palaiseau) gael.latour@polytechnique.edu

$\gg$ Jean-Philippe ECHARD

Laboratoire de recherche et de restauration, Cité de la musique, Paris Centre de recherche sur la conservation des collections (MNHN)

jpechard@cite-musique.fr

L es violons et autres instruments de musique vernis anciens suscitent depuis plus de deux siècles l'admiration, mais aussi de multiples interrogations chez les musiciens, luthiers, scientifiques et amateurs. De nombreux luthiers cherchent aujourd'hui à reproduire les effets optiques de teinte, de chatoyance et de " profondeur " des vernis anciens qui mettent en valeur la structure et les motifs des bois. Cependant, les techniques de vernissage des anciens maîtres-luthiers (jusqu'au XVIII siècle) sont encore relativement peu connues : il n'existe pas de sources écrites directes qui décrivent les matériaux, gestes et procédés utilisés par tel ou tel luthier, aussi célèbre soit-il qu'Antonio Stradivari...

Il est donc nécessaire d'étudier les instruments fabriqués à ces époques et qui nous sont parvenus, afin d'y déceler des informations sur le savoir-faire du luthier en matière de vernis.

Par ailleurs, il peut être précieux au restaurateur d'avoir des informations les plus exhaustives possible afin d'envisager l'intervention la plus adéquate sur de tels objets de notre patrimoine.

\section{Qu'est-ce qu'un vernis ?}

Un vernis peut être simplement décrit comme un milieu stratifié composé de plusieurs couches de vernis appliquées sur le bois de l'instrument, chacune de ces couches (d'épaisseurs variables) ayant sa propre composition (figure 1) et étant susceptible de contenir des particules diffusantes comme les pigments ou des colorants dissous.

Obtenir des informations stratigraphiques est donc crucial pour décrire les techniques anciennes de vernissage. Néanmoins, si elles ne sont accessibles qu'en prélevant un échantillon stratigraphique sur les instruments, ce qui est le cas pour l'instant, les valeurs patrimoniales de ces instruments anciens, en particulier dans des collections de musées, rendent difficiles des prélèvements pour de telles études.

Le musée de la Musique de Paris développe donc depuis 2002 une méthodo- logie d'analyse physico-chimique permettant à la fois de caractériser les matériaux composants ces vernis anciens et leur structure stratigraphique en privilégiant des techniques non destructives [1]. Les techniques optiques y sont donc présentes et porteuses d'un fort potentiel par des analyses sans contact et non destructives, et les techniques d'imagerie y occupent une place de choix !

\section{Un OCT "sur mesure»}

La tomographie par cohérence optique (Optical Coherence Tomography, OCT) a été développée initialement pour l'imagerie biomédicale et y occupe à ce jour une place reconnue avec la présence sur le marché d'appareils commerciaux en ophtalmologie pour l'imagerie de la rétine. Toutefois, son intérêt pour réaliser une biopsie optique sur des tissus biologiques sont transposables à l'étude des objets du patrimoine puisqu'elle permet d'obtenir des images à trois dimensions avec une haute résolution spatiale en réalisant des coupes optiques

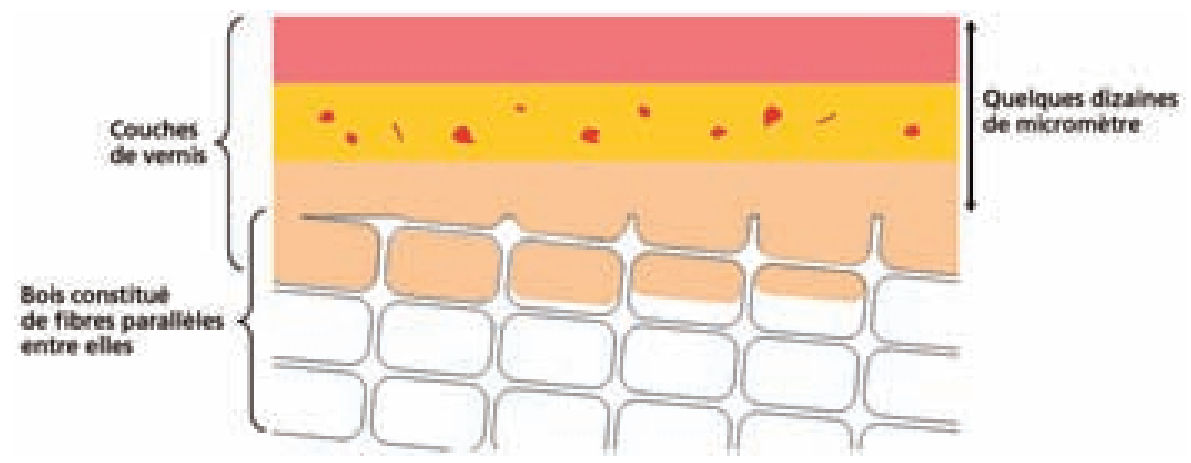

Figure 1. Vue schématique de la stratigraphie d'un vernis présent sur un instrument en bois. 


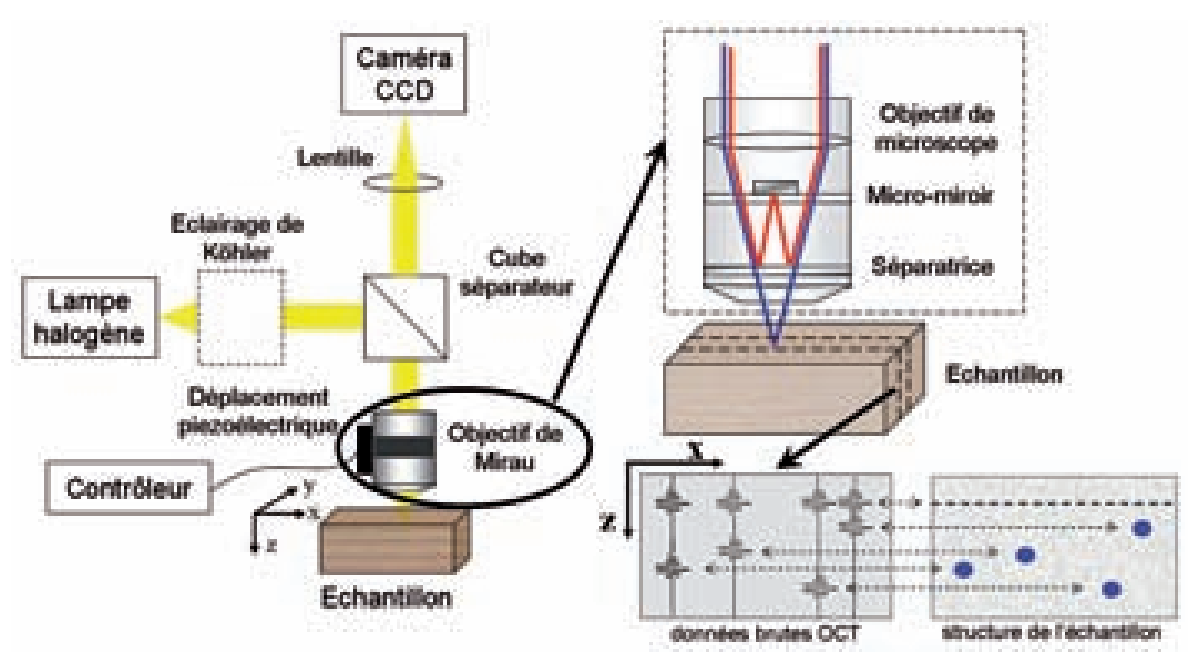

Figure 2. Schéma de principe de I'OCT plein champ développé à I'INSP.

virtuelles à des profondeurs variables. Les photons réfléchis ou diffusés à la profondeur d'intérêt sont sélectionnés à l'aide d'un interféromètre de Michelson dont la figure d'interférences est enregistrée en sortie par un détecteur. En utilisant une source lumineuse présentant une faible cohérence temporelle ou, de manière équivalente, une large bande spectrale, les interférogrammes seront faiblement étalés spatialement et permettent de déterminer précisément les interfaces présentes dans l'objet étudié, sans aucun marquage spécifique des structures que l'on cherche à imager.

Un dispositif expérimental d'OCT plein champ a été développé à l'Institut des nanosciences de Paris (INSP) dans l'équipe Optique et art pour répondre aux contraintes spécifiques liées à l'étude de ces objets uniques (figure 2).

Basé sur le principe d'un interféromètre de Linnik (interféromètre de Michelson avec deux objectifs de microscope), la principale originalité du montage optique développé réside dans l'utilisation d'un objectif interférométrique (également appelé objectif de Mirau), qui intègre dans sa conception le bras de référence de l'interféromètre. Le banc expérimental est ainsi plus compact et plus stable et permet également l'étude d'objets volumineux, tels les instruments de musique car l'objet étudié est fixe et le sondage optique consiste à faire varier la distance entre l'objectif et l'échantillon pour focaliser la lumière à des profondeurs variables.

La source utilisée est une lampe halogène en raison de son bas coût et de son large spectre dans le domaine du visible et du proche infrarouge. Du fait de la sensibilité de la caméra CCD, le dispositif développé est sensible dans le domaine visible. Toute interface ou particule diffusante dans le milieu étudié donne lieu à un interférogramme dû au changement de l'indice de réfraction, et l'image tomographique est obtenue en calculant l'enveloppe des interférogrammes. Les résolutions spatiales atteintes sont de l'ordre du micromètre dans les trois dimensions.

\section{De l'étude du bois...}

La structure du bois (fibres, rayons, parois cellulaires...) est habituellement étudiée en xylologie à partir de coupes minces réalisées selon trois plans bien établis repérés par rapport à l'axe du tronc de l'arbre et observés sous microscopie optique.

L'étude d'un échantillon massif d'érable ondé (bois traditionnellement utilisé pour réaliser le dos, la tête et les éclisses des instruments à cordes en bois) par OCT illustre bien ses possibilités dans l'étude du bois car elle permet d'acquérir des volumes de données. Il est alors aisé de réaliser dans ce volume des coupes virtuelles selon différentes $\gg$

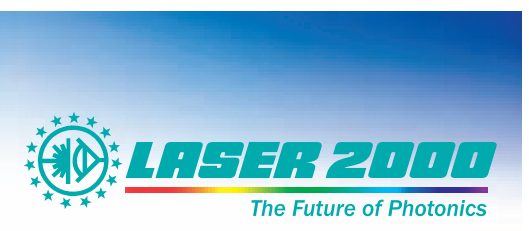

\section{Instrumentations et Mesures}

- Sphères intégrantes, Métrologie de la lumière

- Photomètre Radiomètre et Colorimètre

- Caméras hautes performances

- Monochromateurs et Spectrofluorimètres

- Mesure spectrale

- Analyseurs de faisceaux

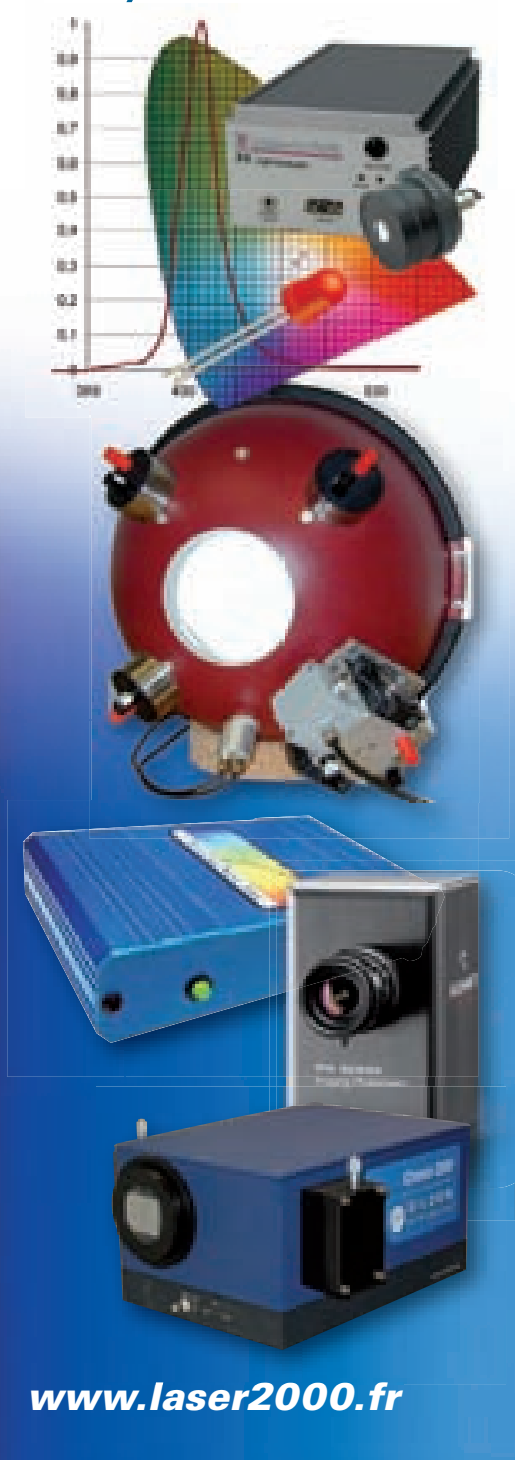


orientations correspondant aux images de microscopie optique utilisées par les professionnels du bois (figure 3 ).

$E n O C T$, seules les interfaces réfléchissantes ou diffusantes donnent lieu à un signal et révèlent donc des structures qui peuvent différer de celles visualisées en microscopie optique. Néanmoins, les images sont très prometteuses, car elles sont notamment porteuses d'informations dans le volume de l'échantillon. L'OCT se révèle aussi une technique de choix pour l'étude des films de vernis, peu diffusants et faiblement absorbants dans la gamme spectrale de notre système $\mathrm{OCT}$, car elle permet de déterminer la présence de plusieurs couches stratifiées, d'évaluer l'épaisseur de ces couches et enfin d'imager la présence éventuelles de particules diffusantes [2].

\section{... à l'étude d'un violon}

Fort de cette expérience d'imagerie OCT sur le bois et ses finitions, nous avons pu envisager l'étude d'un violon italien du XVIII ${ }^{\mathrm{e}}$ siècle du luthier milanais Petro Antonio Landolfi (figure 4).
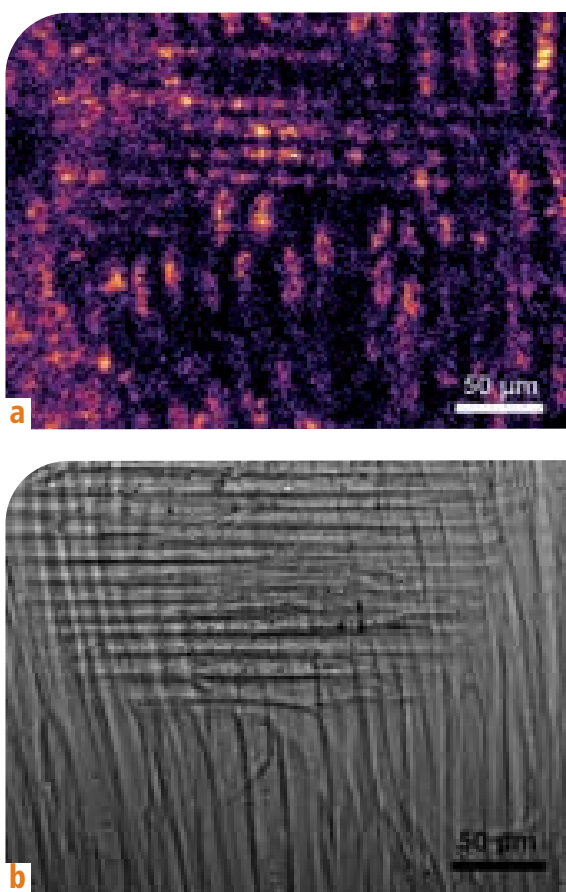

Figure 3. Coupe tomographique (a) d'un échantillon d'érable ondé - particulièrement prisé des luthiers pour son aspect visuel particulier constitué d'une succession de zones sombres et claires - comparée à l'image de microscopie optique (b) d'un prélèvement de ce même échantillon.
Le luthier en charge de l'instrument supposait la présence d'un vernis superficiel déposé au cours d'une restauration au $X I X{ }^{e}$ siècle qui donnait à l'instrument une couleur rouge prononcée. Et, en effet, les mesures OCT ont permis de cartographier la présence et l'épaisseur de ce vernis non original en différentes zones de la table et du dos du violon, mais aussi d'y découvrir la présence de charges (figure 5).

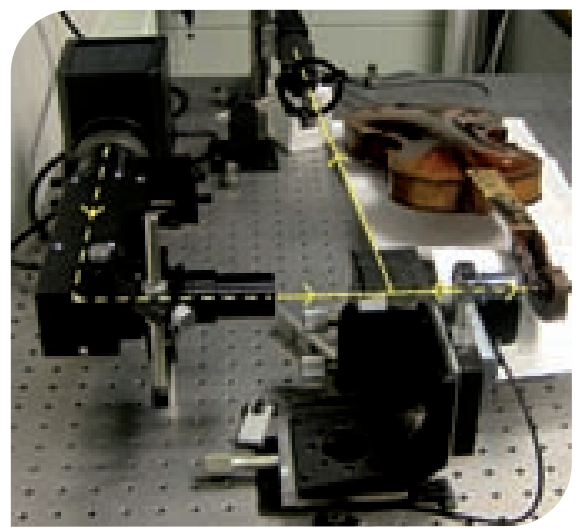

Figure 4. Etude in situ d'un violon du xvIII (Landolfi, Milan, Italie, collection privée) par OCT plein champ. Le trajet optique du faisceau est représenté en jaune.
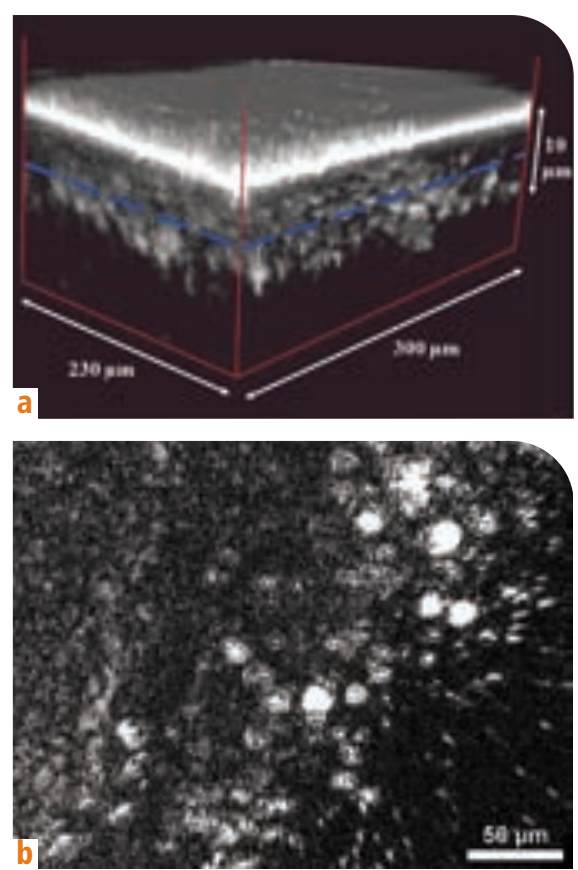

Figure 5. Les images tomographiques de la table du violon ont permis de déceler la présence d'un vernis superficiel supposé non original (datant probablement du xIXe siècle), d'en déterminer l'épaisseur en différentes zones (a) et d'y déceler sur les coupes transverses (b) la présence de particules diffusantes, vraisemblablement des pigments.
On peut dès lors faire l'hypothèse que ces particules diffusantes sont des pigments qui donnent sa couleur au vernis [2].

De telles informations sont précieuses pour les professionnels du patrimoine d'autant qu'elles sont obtenues sans aucun prélèvement de matière.

\section{Vers une information spectrale...}

L'imagerie structurale d'un objet du patrimoine apporte des renseignements utiles. Cependant, la coloration, fréquente, des vernis participe à l'attrait visuel de l'instrument et il serait donc très intéressant de pouvoir disposer, en complément des coupes virtuelles, d'une information liée à la couleur. Une première étude réalisée à l'Institut Fresnel, dans I'équipe Milieux aléatoires et photonique de puissance (MAP2), a montré qu'il est possible de combiner ces informations [3] en complétant le dispositif expérimental précédent avec une roue à filtres à la sortie du dispositif d'illumination pour travailler dans des bandes spectrales distinctes. L'utilisation de ces bandes spectrales dégrade la résolution axiale au niveau de I'imagerie, mais cette technique permet d'ouvrir la voie à une imagerie tridimensionnelle multispectrale.

Plus généralement, dans le cadre d'études sur des objets du patrimoine, I'OCT peut s'avérer être un outil complémentaire d'autres techniques d'analyse in situ telles que la spectrométrie de fluorescence $X$ (EDXRF) ou la spectrofluorimétrie. Du fait de l'aspect sans contact et non destructif et du caractère tridimensionnel des analyses réalisées, il devient donc possible d'accéder à une information en volume et ce en un grand nombre de zones d'analyses, réparties sur l'ensemble de l'objet d'art.

\section{Références :}

[1] Echard J.-P. et al., Angew. Chem. Int. Ed. 49, 197201 (2010).

[2] Latour G. et al., Appl. Opt. 48, 6485-6491 (2009).

[3] Latour G. et al., Proc. SPIE 7391, 73910J (2009). 